\title{
Spectral Demodulation System Based on Two Transmission Volume Bragg Gratings
}

\author{
Guanghui SUI ${ }^{1,2}$, Zhiqi WANG ${ }^{3}$, Ning $\mathrm{WANG}^{3 *}$, \\ Peijian $\mathrm{HUANG}^{3}$, Yong $\mathrm{ZHU}^{3}$, and Jie $\mathrm{ZHANG}^{3}$ \\ ${ }^{1}$ School of Optics and Photonics, Beijing Institute of Technology, Beijing 100081, China \\ ${ }^{2}$ Changcheng Institute of Metrology and Measurement, Beijing 100095, China \\ ${ }^{3}$ The key Laboratory of Optoelectronic Technology \& System (Ministry of Education), Chongqing University, Chongqing \\ 400044, China \\ *Corresponding author: Ning WANG \\ Email: ningw@cqu.edu.cn
}

\begin{abstract}
In this paper, a fiber grating demodulation system based on two transmission volume Bragg gratings (VBGs) was proposed. In order to resolve the problem that the spectral resolution of the present fiber grating demodulation system is not high enough, the two transmission VBGs were applied to improve the spectral resolution and reduce the volume of the spectrometer. The diffraction characteristics of the transmission VBGs were analyzed, and the optical path of the two transmission VBGs demodulation system was designed based on the diffraction characteristics. The grating constant, lens parameters, and aberration correction of the system were analyzed and calculated. The calculation showed that the theoretical wavelength range of the demodulation system was from $1525 \mathrm{~nm}$ to $1565 \mathrm{~nm}$ and the theoretical optical resolution was $60 \mathrm{pm}$ when the grating constant was 0.9168 , the angle between two transmission VBGs was $89^{\circ}$, the focal length of the collimator was $60 \mathrm{~mm}$, and the focal length of the imaging lens was $131.5 \mathrm{~mm}$. The aberration of the system was well corrected by using a lens as the collimator and a reflector as the imaging lens. The system principle prototype was assembled and calibrated, and its performances were experimentally investigated. The results showed that the spectrometer worked stably, with a wavelength range from $1525 \mathrm{~nm}$ to $1565 \mathrm{~nm}$, an optical wavelength resolution of $65.3 \mathrm{pm}$, and a high demodulation speed of $10 \mathrm{kHz}$.
\end{abstract}

Keywords: Spectrometer; diffraction; transmission volume Bragg grating; fiber grating

Citation: Guanghui SUI, Zhiqi WANG, Ning WANG, Peijian HUANG, Yong ZHU, and Jie ZHANG, "Spectral Demodulation System Based on Two Transmission Volume Bragg Gratings," Photonic Sensors, 2021, 11(3): 334-340.

\section{Introduction}

In the past few decades of fast-growing aerospace industry, the aero-engine has been addressed as one of the most important engineering problems in the national defense and civil aviation safety. It is of great significance in monitoring structural state of aero-engine [1]. Conventional electric sensors can hardly meet the needs of in-situ measurement of the aero-engine because it is limited by the low measurement temperature and large volume. The fiber sensor has the potential for the in-situ measurement of the aero-engine, with its advantages of the small size, high temperature

Received: 12 September 2019 / Revised: 23 June 2020

(C) The Author(s) 2020. This article is published with open access at Springerlink.com

DOI: $10.1007 / \mathrm{s} 13320-020-0594-1$

Article type: Regular 
resistance, and electromagnetic interference resistance. Recently, many researchers proposed some new fiber sensors to detect strain and temperature under high temperature environments. In 2008, Rao et al. [2] designed the photonic crystal fiber based Fabry-Perot sensor that was insensitive to temperature change within $800{ }^{\circ} \mathrm{C}$, and the sensitivity of strain measurement was $0.32 \mathrm{~nm} / \mu \mathrm{m}$. In 2012, F. C. Favero et al. [3] designed a Fabry-Perot (F-P) sensor by using photonic crystal fiber (PCF) and single-mode fiber fused microcavity. And the strain sensitivity of the sensor could reach $10.3 \mathrm{pm} / \mu \varepsilon$. In 2015, Liu et al. [4] proposed F-P sensors based on in-fiber reshaped air bubbles with $6 \mathrm{pm} / \mu \varepsilon$ of the elliptical bubble and $43 \mathrm{pm} / \mu \varepsilon$ of the rectangular bubble, and the temperature sensitivity of them was both $2 \mathrm{pm} /{ }^{\circ} \mathrm{C}$. In 2011, Wang et al. [5] found that the fiber Bragg grating (FBG) fabricated by the femtosecond directing writing technology could work stably within $1200^{\circ} \mathrm{C}$. In 2013, Niu et al. [6] proposed an FBG high-temperature strain sensor with the strain sensitivity of $369.4 \mathrm{pm} / \mathrm{N}$ within $250^{\circ} \mathrm{C}$.

The FBG sensor (sapphire) fabricated by the femtosecond direct writing technology is expected to measure the temperature and strain of the aero-engine, with its advantages of high temperature resistance, high pressure resistance, electromagnetic interference resistance, small size, and distributed detection which are easy to be implemented [7-9]. The melting point of the FBG sensor (sapphire) can be up to $2000{ }^{\circ} \mathrm{C}$. And the sensitivities of the FBG sensor to detect the temperature and strain are about $10 \mathrm{pm} /{ }^{\circ} \mathrm{C}$ and $1.2 \mathrm{pm} / \mu \varepsilon$ according to our previous testing result, which are enough to meet the measurement requirements $\left(1800^{\circ} \mathrm{C}\right.$ and $\left.24000 \mu \varepsilon\right)$ of the aero-engine with the wavelength range from $1525 \mathrm{~nm}$ to $1565 \mathrm{~nm}$. Comparing conventional fiber grating demodulation means, the static demodulation methods, such as the matched grating method $[10,11]$ and tunable F-P filter method [12, 13], have a low demodulation speed, which is not suitable for the dynamic real-time monitoring in the aerospace, while the interference method $[14,15]$ is sensitive to the temperature of circumstance. The spectral demodulation method $[16,17]$ is easily and reliably used to measure the central wavelength shift of the reflected light of the fiber grating by a spectrometer. However, they cannot satisfy demodulation demands of high speed $(10 \mathrm{kHz})$, high precision $(\sim 100 \mathrm{pm})$, and volume as small as possible (for airborne), although the resolution of demodulation can be increased by an interpolation fitting. In addition, the sampling rate of some present commercial spectrometers (Ibsen Company) is up to $17 \mathrm{kHz}-35 \mathrm{kHz}$, but the optical wavelength resolution of them is also low.

In this paper, we proposed a new spectral system to demodulate the FBG sensor for increasing the demodulation speed and resolution. The system was based on two transmission volume Bragg gratings (VBGs) and a high-speed InGaAs array detector. The optical characteristics of two transmission VBGs were analyzed, and the external dimensions and parameters of the optical system were optimized. The installation and the system test platform were completed, and the performance of the spectrometer was tested.

\section{Optical structure design}

Figure 1 shows the optical structure, containing a light source, a collimation system, a dispersion system, a spherical reflector, and an InGaAs array photodetector. The light reflected from the FBG sensor was incident on the spectrometer through the fiber coupler. For compacting the structure and avoiding the interference of stray light, a plane reflector tilted at $45^{\circ}$ was used. A cemented doublet lens was adopted to collimate light, and simultaneously to correct spherical aberration and chromatic aberration. Especially, we chose two transmission VBGs as the dispersion system. On the one hand, the VBG possessed a high and flat diffraction efficiency [18], increasing the optical 
resolution of the system. On the other hand, it could reduce the volume of the spectrometer. A spherical reflector was used to focus the beam on the imaging surface of the detector and make imaging quality better, because a mirror doesn't produce chromatic aberration. A high-speed InGaAs array detector was applied to replace the traditional mechanical scanning structure, which increased the demodulation speed.

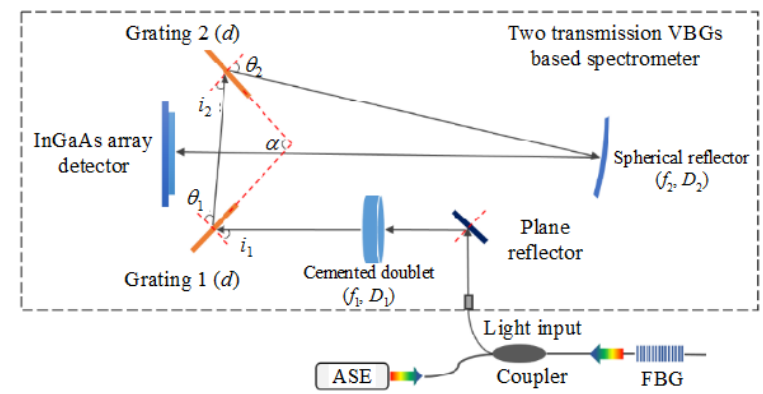

Fig. 1 Optical structure of two transmission VBGs based spectrometer.

We analyze the important characteristics as follows. Firstly, the grating constant $d$ and the angle $\alpha$ between two VBGs can be calculated by the grating equation. For the first grating $G_{1}$, when the incident angle is $i_{1}$, the diffraction angle $\theta_{1}$ can be expressed as

$$
\sin \theta_{1}=\frac{\lambda}{d}-\sin i_{1}
$$

where $\lambda$ is the wavelength of the incident light. The relationship between the incident angle $i_{2}$ of the second grating and the diffraction angle $\theta_{1}$ of the first grating can be expressed as

$$
i_{2}=\alpha-\theta_{1}
$$

where $\alpha$ is the angle between two VBGs.

For the second grating $G_{2}$ similarly, the diffraction angle $\theta_{2}$ can be determined as

$$
\sin \theta_{2}=\frac{\lambda}{d}-\sin \left(\alpha-\theta_{1}\right)<1 .
$$

We can get a one-variable quadratic inequality with respect to $\sin \alpha$ as

$$
\sin ^{2} \alpha-2\left(\frac{\lambda}{d}-1\right) \cos \theta_{1} \sin \alpha+\left(\frac{\lambda}{d}-1\right)^{2}-\sin ^{2} \alpha>0 .
$$

Note that the detection demand of the wavelength range is from $1525 \mathrm{~nm}$ to $1565 \mathrm{~nm}$. The calculated the grating constant $d$ should be larger than 0.9168 , and the grating lines within $1 \mathrm{~mm}$ should be smaller than 1090.8 lines $/ \mathrm{mm}$.

According to the market, we chose the grating with 966 lines/mm (absolute diffraction efficiency of more than $90 \%$ within the wavelength range from $1525 \mathrm{~nm}$ to $1565 \mathrm{~nm}$ ), and the detector of 2048 pixels (pixel rate of $25 \mathrm{MHz}$ and line rate of $10 \mathrm{kHz}$ ) with the pixel resolution of $40 \mathrm{~nm} / 2048$.

The solution of (4) is that $\alpha$ should be larger than $85.16^{\circ}$, according to (1) and (3) when $i_{1}=45^{\circ}$, $\lambda=1565 \mathrm{~nm}$, and $d=1 \mathrm{~mm} / 966$. The angle $\alpha$ between two VBGs is as large as possible to make sure that there is enough emergent light after the glass base is put on the grating. However, an oversize angle will lead to the looseness of the structure and a poor imaging quality. Therefore, the angle $\alpha$ between two VBGs was determined to be $89^{\circ}$ on the basis of the numerical calculation.

Secondly, to match the imaging size with the actual dimension of the InGaAs array detector, the numerical calculation showed that the whole imaging size was $25.8 \mathrm{~mm}$, the radius of the spherical reflector was $263 \mathrm{~mm}$, and the focal length $f_{2}$ was $131.5 \mathrm{~mm}$. The clear aperture and outer diameter of the spherical reflector were $30 \mathrm{~mm}$ and $34 \mathrm{~mm}$, respectively. And there was $4 \mathrm{~mm}$ redundant width of the outer diameter for clamping the lens. We chose the width of $12 \mathrm{~mm}$ in the middle part of the spherical reflector for imaging with better quality, because the light would not reach the edge of the mirror.

Thirdly, the angular dispersion and linear dispersion were analyzed. By differentiating (3) with respect to $\lambda$, we obtain

$$
\frac{\mathrm{d} \theta_{2}}{\mathrm{~d} \lambda}=\frac{1}{d \cos \theta_{2}}\left[1+\cos \alpha+\frac{\sin \alpha\left(\frac{\lambda}{d}-\sin i_{1}\right)}{\sqrt{1-\left(\frac{\lambda}{d}-\sin i_{1}\right)^{2}}}\right] .
$$

The diffraction angle difference is calculated to 
be $10.59^{\circ}$, when the angle $\alpha$ is $89^{\circ}$, the angle $i_{1}$ of the incident ray is $45^{\circ}$, and the upper limit $\lambda_{1}$ and the lower limit $\lambda_{2}$ of the wavelength are $1525 \mathrm{~nm}$ and $1565 \mathrm{~nm}$, respectively, whereas the diffraction angle difference of the spectrometer based on a single grating is $3.58^{\circ}$ with the same conditions. Our design can increase the optical resolution, as well as reduce the volume of the spectrometer.

Meanwhile, the linear dispersion $\Delta l$ can be derived as

$\Delta l=\int_{\lambda}^{\lambda+\Delta \lambda} \frac{1}{d \cos \theta_{2}}\left[1+\cos \alpha+\frac{\sin \alpha\left(\frac{\lambda}{d}-\sin i_{1}\right)}{\sqrt{1-\left(\frac{\lambda}{d}-\sin i_{1}\right)^{2}}}\right] f_{2} \mathrm{~d} \lambda$.

For the central wavelength $\lambda$ of $1545 \mathrm{~nm}$, when the resolution $\Delta \lambda$ is $60 \mathrm{pm}$ and the focal length $f_{2}$ of imaging lens is $131.5 \mathrm{~mm}$, the slit image is calculated to be $35.79 \mu \mathrm{m}$. The amplification $\beta$ of the vertical axis can be expressed as

$$
\beta=\frac{\Delta l}{y}=\frac{l^{\prime}}{l}=\frac{f_{2}}{f_{1}^{\prime}}
$$

where $y$ is the silt width of $10 \mu \mathrm{m}, l$ is the object intercept, $l^{\prime}$ is the image intercept, and $f_{1}^{\prime}$ is the collimator focal length. The focal length $f_{1}$ of the cemented doublet is calculated to be $36.74 \mathrm{~mm}$. We can increase the focal length $f_{1}$ properly while others remain unchanged, which can lead to a smaller slit imaging $\Delta l$. Through optimized numerical calculations, the focal length $f_{1}$ is determined to be $60 \mathrm{~mm}$ for meeting the demands of imaging and easily being processed.

$$
\tau=\frac{\lambda_{2}-\lambda_{1}}{n}
$$

where the pixel number $n$ of the InGaAs detector array is 2048 , and $\lambda_{1}$ and $\lambda_{2}$ are $1525 \mathrm{~nm}$ and $1565 \mathrm{~nm}$, respectively. So the pixel resolution $\tau$ is about $0.02 \mathrm{~nm}$. We know that one spectral line can be identified by three separated pixels. Therefore, the optical wavelength resolution $\Delta \lambda$ of this system is $60 \mathrm{pm}$.
According to the half angular width of line and angular dispersion, the color dispersion of two transmission VBGs is derived as

$$
\frac{\lambda}{\Delta \lambda}=N\left[1+\frac{\left(\frac{\lambda}{d}-\sin i\right)}{\sqrt{1-\left(\frac{\lambda}{d}-\sin i\right)^{2}}}\right]
$$

where $N$ is the total number of grating lines within the light area. The light spot size $L$ of the grating can be determined to be $11.75 \mathrm{~mm}$, when $\lambda$ is $1545 \mathrm{~nm}$, the resolution $\Delta \lambda$ is $60 \mathrm{pm}$, the incident angle $i$ is $45^{\circ}$, and the grating constant $d$ with the unit irradiation area is $1 \mathrm{~mm} / 966$. Considering the existing of oblique incidence of $45^{\circ}$ angle in this system, the manufacture error, and clamping allowance of the lens, the final clear aperture and outer diameter of cemented doublet are determined to be $9 \mathrm{~mm}$ and $11 \mathrm{~mm}$, respectively.

The overall optimized structure of the system in ZEMAX is shown in Fig. 2.

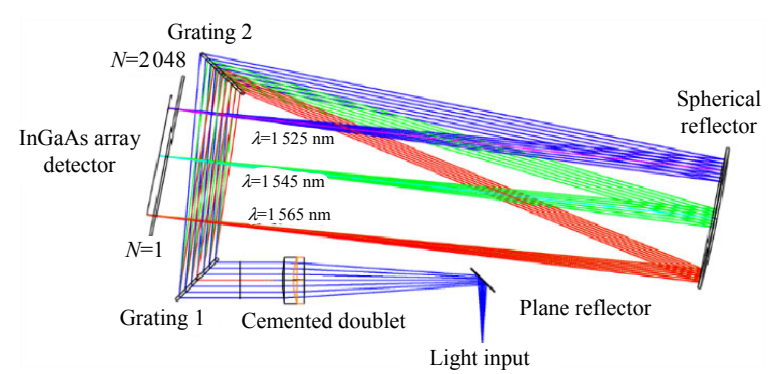

Fig. 2 System simulation of two transmission VBGs based spectrometer.

For better comparison with the single-grating based spectrometer, Fig. 3 shows the relationship between the diffraction angle difference $\Delta \theta_{2}$ and the focal length $f_{2}$ of the spherical reflector. It can be expressed as

$$
f_{2}=\frac{L}{2 \tan \frac{\Delta \theta_{2}}{2}} .
$$

When $L=25.8 \mathrm{~mm}$, the diffraction angle differences $\Delta \theta_{2}$ of two-grating system and single grating system are $10.59^{\circ}$ and $3.58^{\circ}$, respectively. If we maintain the same pixel resolution, the calculated distances $f_{2}$ from the spherical reflector to 
the detector is calculated to be $139 \mathrm{~mm}$ (two-grating system) and $412 \mathrm{~mm}$ (single-grating system), respectively. This indicates that the two-grating system has smaller volume than the single grating system.

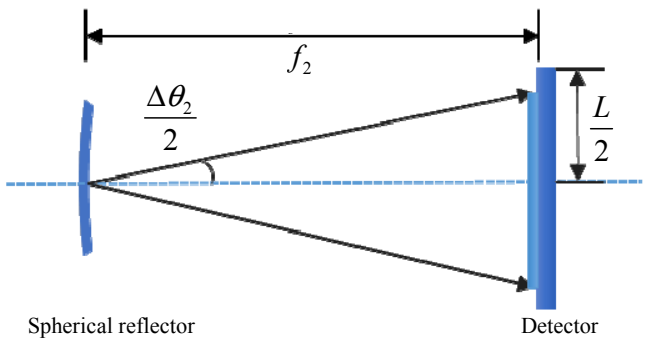

Fig. 3 Relationship between the focal length $f_{2}$ and the diffraction angle difference $\Delta \theta_{2}$.

\section{Results and discussion}

Three typical wavelengths with $1525 \mathrm{~nm}$, $1565 \mathrm{~nm}$, and $1545 \mathrm{~nm}$ were used to verify the optical resolution of the system. Figure 4 shows three groups of light spots with wavelength difference of $60 \mathrm{pm}$ on the image plane. Based on Rayleigh criterion, the overlapping of light spots of $1525 \mathrm{~nm}$ and $1525.06 \mathrm{~nm}$ was $0.017 \mathrm{~mm}$, less than $d_{1} / 2\left(d_{1}\right.$ is the diameter of the slit image, as shown in Fig.4), which proved the two light spots could be clearly distinguished. Similarly, the light spots at $1545 \mathrm{~nm}$ and $1565 \mathrm{~nm}$ could be resolved as well. Therefore, the resolution of two transmission VBGs based spectrometer met the demand of $60 \mathrm{pm}$.

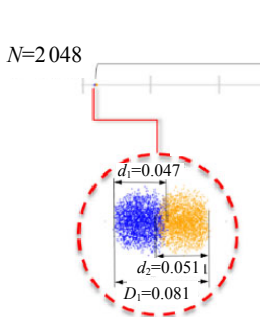

(a)
Detector used

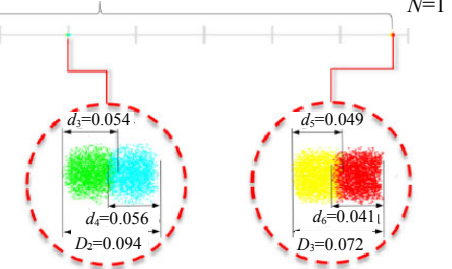

(b)

(c)
Fig. 4 Three sets of image spots in different wavelength ranges: (a) $1525 \mathrm{~nm}$ (blue) and $1525.06 \mathrm{~nm}$ (orange), (b) $1545 \mathrm{~nm}$ (green) and $1545.06 \mathrm{~nm} \mathrm{(sky} \mathrm{blue),} \mathrm{and}$ (c) $1565 \mathrm{~nm}$ (red) and $1564.94 \mathrm{~nm}$ (yellow).

The assembly of two transmission VBGs based spectrometer was successfully completed. The final locations of the lens and detector were fine-tuned according to the imaging results on the spectral plane. Finally, they were solidified by using the silicone rubber after adjustment, as shown in Figs. 5(a) and 5(b).

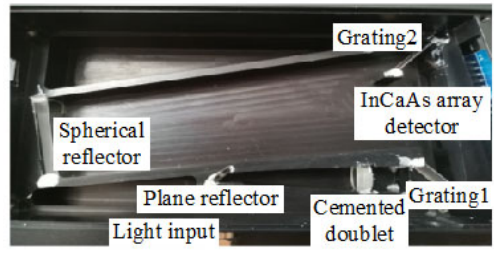

(a)

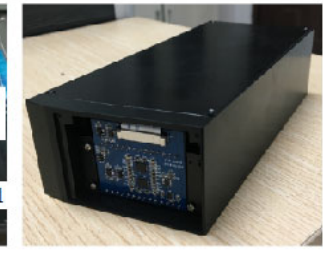

(b)
Fig. 5 Actual structure of the spectrometer: (a) optical structure and (b) side view of the spectrometer.

For measurements of the actual wavelength range and resolution of our system, the tunable laser AQ4321D was chosen as the light source $(1520 \mathrm{~nm}$ to $1570 \mathrm{~nm}$ ). It was scanned at a step of $0.01 \mathrm{~nm}$ with an output power of $1 \mathrm{~mW}$. We took three typical wavelengths as testing examples.

Figures 6(a) and 6(b) show that the wavelength of $1525 \mathrm{~nm}$ is located at the 2026th pixel of the detector, while $1565 \mathrm{~nm}$ is corresponding to 29th pixel. In the figures, the value of the $y$-axis is the sample value of the analog-to-digital converter (ADC). Our system fulfilled the design requirement of the wavelength range. The pixel resolution $\tau$ was $0.02003 \mathrm{~nm}$ according to (8). The calibration results of the intermediate wavelength $1545 \mathrm{~nm}$ are shown in Fig. 6(c). There were 3.26 pixels included in the full width at half maximum (FWHM) of the spectral lines. So, the actual resolution of the spectrometer was $65.3 \mathrm{pm}[(0.02003 \times 3.26) \mathrm{pm}]$.

In addition, the light efficiency $(\sim 47.2 \%)$ of the spectral system depended on the loss of fiber transportation and coupling $(\sim 25 \%)$, the loss of the cemented doublet $(\sim 1 \%)$, the loss of the spherical mirror $(\sim 1 \%)$ and the plane mirror $(\sim 1 \%)$, and the efficiency of the VBGs (>90\%) and InGaAs detector $(\sim 80 \%)$. We also took measurement for a signal with a period of $100 \mu \mathrm{s}$, and our system could clearly detect it, indicating the modulation speed was $10 \mathrm{kHz}$ 


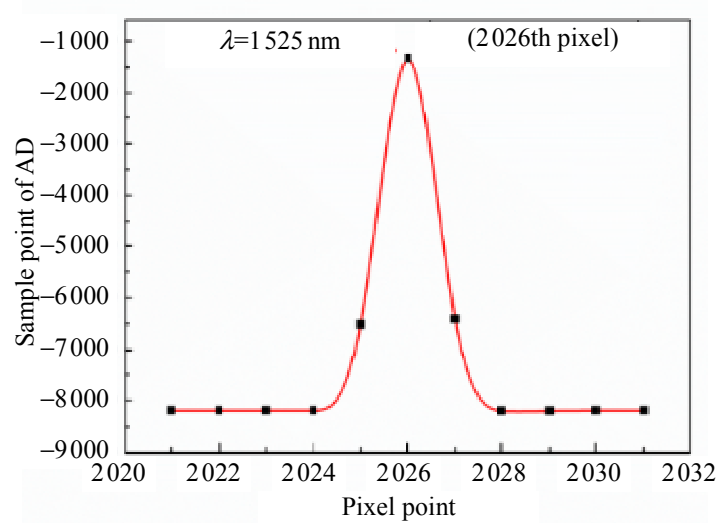

(a)

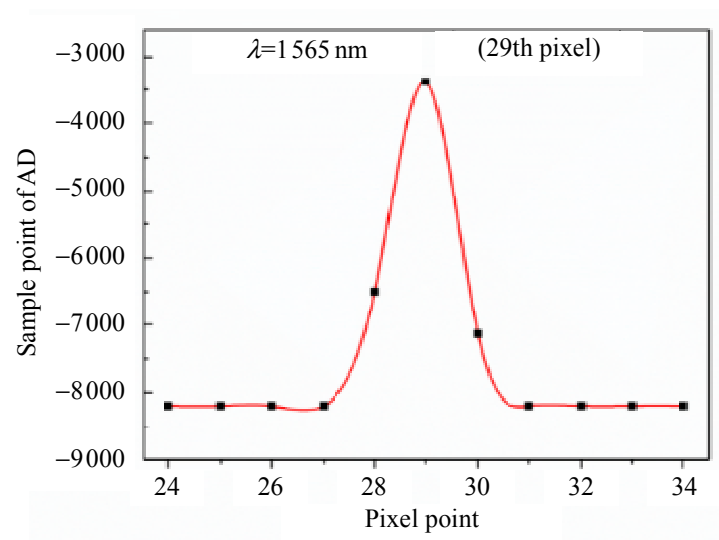

(b)

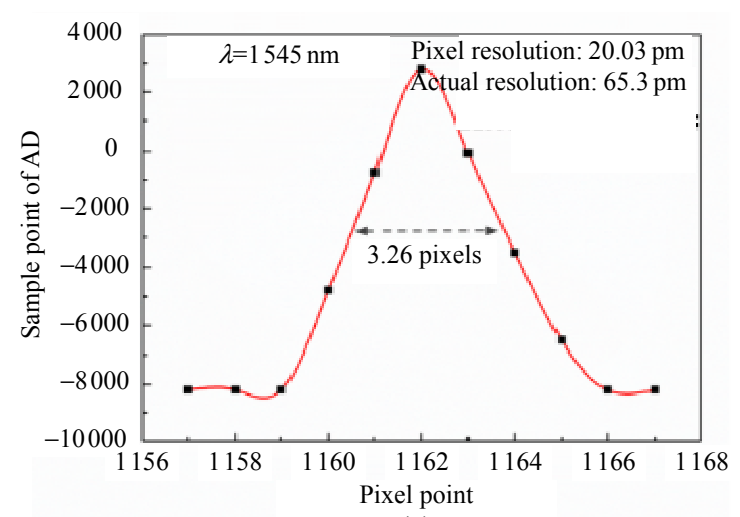

(c)

Fig. 6 Calibration results of the spectrometer: (a) the pixel position corresponding to $1525 \mathrm{~nm}$, (b) the pixel position corresponding to $1565 \mathrm{~nm}$, and (c) the pixel position corresponding to $1545 \mathrm{~nm}$.

\section{Conclusions}

In conclusion, our two transmission VBGs based spectrometer could work well in the wavelength range from $1525 \mathrm{~nm}$ to $1565 \mathrm{~nm}$, with the optical wavelength resolution of $65.3 \mathrm{pm}$ and demodulation speed of $10 \mathrm{kHz}$. Especially, the diffraction angle difference was $10.59^{\circ}$, much larger than that $\left(3.58^{\circ}\right)$ of the traditional single grating based spectrometer, hence reducing the volume of the spectrometer. It can be used to demodulate the signal of fiber grating sensors, with specially high speed and high precision.

\section{Acknowledgment}

This work was supported by the National Natural Science Foundation of China (Grant No. 51875067) and the Fundamental Research Funds of Central Universities (Grant No. 2019CDJGFGD003).

Open Access This article is distributed under the terms of the Creative Commons Attribution 4.0 International License (http://creativecommons.org/licenses/by/4.0/), which permits unrestricted use, distribution, and reproduction in any medium, provided you give appropriate credit to the original author(s) and the source, provide a link to the Creative Commons license, and indicate if changes were made.

\section{References}

[1] T. G. Liu, S. Wang, J. F. Jiang, K. Liu, and J. D. Yin, "Research progress of optical fiber sensing technology in aerospace," Chinese Journal of Scientific Instrument, 2014, 35(8): 1681-1692.

[2] H. Y. Deng, Y. J. Rao, Z. L. Ran, X. Liao, and W. J. Liu, "Photonic crystal fiber based Fabry-Perot sensor fabricated by using $157 \mathrm{~nm}$ laser micromachining," Acta Optica Sinica, 2008, 28(2): 255-258.

[3] F. C. Favero, L. Araujo, G. Bouwmans, V. Finazzi, J. Villatoro, and V. Pruneri, "Spheroidal Fabry-Perot microcavities in optical fibers for high-sensitivity sensing," Optics Express, 2012, 20(7): 7112-7118.

[4] S. Liu, Y. P. Wang, C. R. Liao, Z. Y. Li, and K. M. Yang, et al. "High-sensitivity strain sensors based on in-fiber reshaped air bubbles," in Fifth Asia Pacific Optical Sensors Conference, Korea, May 20-22, 2015, pp. 96550A-1-96550A-4.

[5] Y. H. Li, M. W. Yang, C. R. Liao, D. N. Wang, J. $\mathrm{Lu}$, and P. X. Lu, "Prestressed fiber Bragg grating with high temperature stability," Journal of Lightwave Technology, 2011, 29(10): 1555-1559.

[6] W. Q. Niu, Y. Y. Ma, Y. Wu, Z. L. Yuan, Y. Gong, and Y. J. Rao, "Non-gel encapsulation process of a high temperature strain fiber Bragg grating sensor and its sensing properties," Chinese Journal of Sensors and Actuators, 2013, 26(7): 927-931. 
[7] X. R. Dong, Z. Xie, Y. X. Song, K. Yin, D. K. Chu, and J. A. Duan, "High temperature-sensitivity sensor based on long period fiber grating inscribed with femtosecond laser transversal-scanning method," Chinese Optics Letters, 2017, 15(9): 51-55.

[8] H. Zhang, J. Z. Jiang, S. Liu, H. X. Chen, X. Q. Zheng, and Y. S. Qiu, "Overlap spectrum fiber Bragg grating sensor based on light power demodulation," Sensors, 2018, 18(5): 1597-1-1597-11.

[9] D. Grobnic, S. J. Mihailov, C. W. Smelser, and H. M. Ding, "Sapphire fiber Bragg grating sensor made using femtosecond laser radiation for ultrahigh temperature applications," IEEE Photonics Technology Letters, 2004, 16(11): 2505-2507.

[10] L. A. Ferreira, J. L. Santos, and F. Farahi, "Pseudoheterodyne demodulation technique for fiber Bragg grating sensors using two matched gratings," IEEE Photonics Technology Letters, 1997, 9(4): 487-489.

[11] B. Q. Jiang, J. L. Zhao, C. Qin, and Z. Huang, and F. Fan. "An optimized strain demodulation method based on dynamic double matched fiber Bragg grating filtering," Optics and Lasers in Engineering, 2011, 49(3): 415-418.

[12] Z. A. Jia, J. Liu, X. G. Qiao, T. Wei, H. Gao, and H. F. Feng, "A demodulation technology based on digital tunable F-P filter for fiber Bragg grating sensing signals," Journal of Optoelectronics. Laser,
2011, 5(5): 649-651.

[13] Q. Y. Li, Y. L. Xiong, Y. Xia, M. Z. Wu, J. Y. Zou, and Z. Y. Ma, "The simulation of FBG demodulation system based on the tunable F-P filter," in Proceedings of 2013 2nd International Conference on Measurement, Information and Control, China, Aug. 16-18, 2013, pp. 337-340.

[14] M. A. Davis and A. D. Kersey, "All-fibre Bragg grating strain-sensor demodulation technique using a wavelength division coupler," Electronics Letters, 1994, 30(1): 75-77.

[15] Y. L. Yu, H. Y. Tam, and W. H. Chung, "A fiber Bragg grating sensor system with interferometric demodulation technique," Acta Optica Sinca, 2001, 21(8): 987-989.

[16] D. D. Pang, Q. M. Sui, and M. S. Jiang, "New fiber Bragg grating high temperature sensing network based on diffraction demodulation," Chinese Journal of Lasers, 2011, 38(11): 174-179.

[17] Z. W. Feng and L. Zhang, "Demodulation technique based on diffraction optical elements for fiber Bragg grating sensing system," in Photonics Asia 2010, China, Oct. 17-20, 2010, pp: 78530I-178530I-9.

[18] I. V. Ciapurin, L. B. Glebov, and V. I. Smirnov, "Spectral combining of high-power fiber laser beams using Bragg grating in PTR glass," in Lasers and Applications in Science and Engineering, United States, Jan. 25-29, 2004, pp. 116-124. 